\title{
Putting super-resolution fluorescence microscopy to work
}

\author{
Jennifer Lippincott-Schwartz \& Suliana Manley
}

\begin{abstract}
Super-resolution microscopy is poised to revolutionize our understanding of the workings of the cell. But the technology still has some limitations, and these must be taken into consideration if widespread application is to yield biological insight.
\end{abstract}

Light microscopy has been a favorite tool of biologists for unlocking life's mysteries since Leeuwenhoek first focused light through a lens to study living microorganisms, or "animalcules," in the seventeenth century. Because of Leeuwenhoek's work and that of his successors, we know much about the morphological intricacies of subcellular organization. Since then, there has been a constant search for microscopy techniques to resolve ever-smaller working parts of the cell, culminating today in Nature Methods' technique of the year, super-resolution (SR) imaging, which captures biological processes at the scale of single molecules.

The path to today's SR technology has proceeded in stages. First, fluorescent tagging made it possible to detect specific cellular constituents. By generating antibodies and later proteins that fluoresce, it became possible to noninvasively image the interior of living cells. However, these tools faced a limit because light is imperfectly focused by a microscope lens, and because each object blurs into a spot having a diffraction-limited minimum size, much like the unreadable letters on the lowest line

Jennifer Lippincott-Schwartz and Suliana Manley are in the Cell Biology and Metabolism Program, National Institute of Child Health and Human Development, National Institutes of Health, Building 18T, Room 101, 18 Library Drive, Bethesda, Maryland 20892, USA. e-mail: lippincj@mail.nih.gov or manleysu@mail.nih.gov

PUBLISHED ONLINE 17 DECEMBER 2008; DOI:10.1038/NMETH.F.233

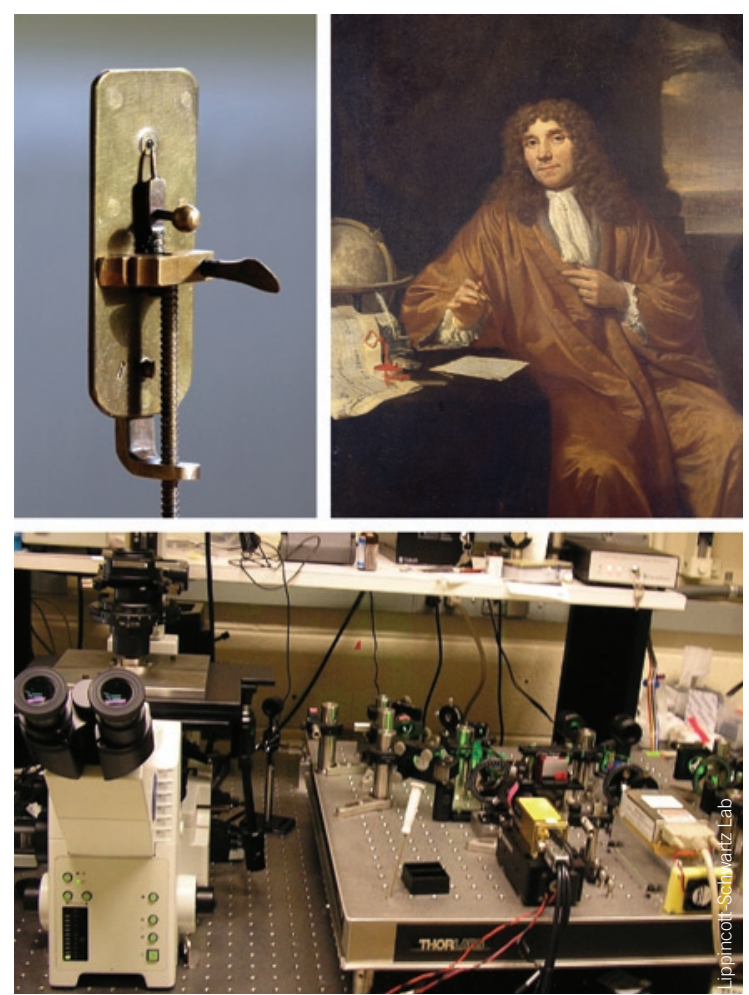

Leeuwenhoek and his first microscope, compared with a nextgeneration instrument for SR PALM imaging.

of an eye chart. Nothing below a quarter of a micrometer could be resolved, yet many cellular structures are much smaller.

This challenge was met initially by using not a lens but rather a very small aperture, positioned close enough to the sample that light does not have a chance to substantially diffract. This 'near-field microscopy' produced nanometer-scale images of molecules ${ }^{1}$ and thus was the first generation of SR technique. It had limitations, however, because only the surface of a sample could be imaged and effective apertures were difficult to make. These problems were overcome by returning to the classical microscope design of placing lenses at a distance from the sample (that is, 'far-field microscopy') but using nonlinear optical approaches to reduce the focal spot size. Among the so-called illumination-based SR imaging techniques are stimulated emission depletion (STED) microscopy ${ }^{2}$ and saturated structured illumination microscopy (SSIM) ${ }^{3}$. More recently, what is called probebased SR imaging has been achieved using photoactivation localization microscopy (PALM) ${ }^{4}$ and the related techniques stochastic optical reconstruction microscopy (STORM $)^{5}$ and FPALM (fluorescence PALM ${ }^{6}$ ) which exploit the stochastic activation of fluorescence. In this probe-based SR imaging, multiple raw images are acquired. In each image, only some of the tagged molecules in the cell are made to fluoresce (that is, they are photoactivated and then excited) and then 'bleached' or switched off to permit imaging of other fluorescing molecules subsequently. Because only a sparse subset of fluorophores is activated during each photoactivation cycle, molecules are localized in the absence of interference from neighboring fluorescent molecules. The final super-resolution image is then constructed by superimposing or merging all the single molecule positions. Structures labeled by an ensemble of photoactivatable fluorescent proteins too 
Super-resolution fluorescence microscopy

STED, SSIM, PALM, STORM

Obstacles

Probe-related: aggregation, mistargeting, signal/noise, specificity

Sample-related: fixation, permeabilization, photodamage

$\downarrow$

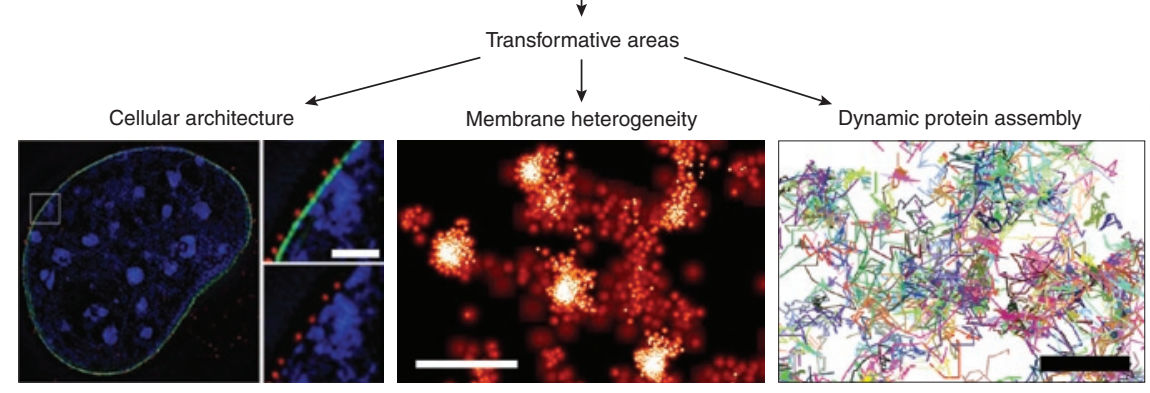

SR techniques, obstacles and transformative areas. Scale bars, $1 \mu \mathrm{m}$. Portions of the figure were reprinted with permission from AAAS.

dense to be imaged simultaneously can

thereby be resolved with nanometric precision and at unprecedented molecular densities (up to $10^{5}$ molecules per $\mu \mathrm{m}^{2}$ ).

What this means is that biologists can now visualize the structures and processes of the cell at the molecular level. Using illumination-based SR approaches, the three-dimensional organization of distinct nuclear pore complex components has been mapped $^{7}$, and protein clusters on individual synaptic vesicles ${ }^{8}$ and in synaptic active zones ${ }^{9}$ have been resolved at the nanometer scale. At the same time, probe-based SR approaches have permitted visualization of the single molecule distribution of proteins on diverse structures such as lysosomes ${ }^{4}$, Golgi apparatus ${ }^{4}$, microtubules ${ }^{10}$ and clathrin-coated vesicles ${ }^{10}$ with $20-30 \mathrm{~nm}$ resolution. Dynamic processes have also been revealed using illumination- or probebased SR imaging, including remodeling of focal adhesions ${ }^{11}$, movement of synaptic vesicles ${ }^{7}$, treadmilling of a bacterial actin-related protein ${ }^{12}$ and the brownian movement of large populations of single molecules ${ }^{13}$.

Nevertheless, SR methodology must still prove itself to biologists as a reliable technique for achieving new biological insight. This will require correlating its results with those from complementary approaches, applying it with cognizance of its particular strengths and weaknesses, and developing standardized guidelines for interpreting SR data.

Because our ideas of molecular organization and dynamics are mainly in the form of conceptual cartoons, observations at the nanoscopic level may reveal things that ini- tially make little sense. For example, there was enormous skepticism when electron microscopy first revealed the dense filamentous meshwork comprising the cytoskeleton. Cell biologists holding the view of cytoplasm as a dilute biochemical soup called the observations a fixation artifact. Unless the new discoveries by SR imaging are supported by complementary data obtained from other techniques, similar skepticism can be expected. Early electron microscopic discoveries accomplished this with supporting evidence from biochemical assays. Unorthodox SR microscopy discoveries will also need support from biochemistry, as well from electron microscopy. The latter is especially important as it provides the needed nanometer-scale resolution of cell ultrastructure to correlate with SR images. Hence, as the focus of SR microscopy shifts from novelty to biological application, careful controls using correlative electron microscopy and other strategies must be adopted to ensure the results are accurate.

Users of SR microscopy must also be sensitive to its current shortcomings. For example, different types of fluorescent probes carry trade-offs in their suitability for SR imaging. Fluorescent proteins can be genetically fused to any cloned cDNA, providing molecular specificity, but when overexpressed can aggregate or lead to mistargeting. Their distribution thus must be compared with that of functional untagged proteins or to that found in more weakly expressing transfected cells. Similarly, cellular autofluorescence or fluorescence from unactivated fluorophores can obscure the signal from single molecules, making sparse- ly labeled structures difficult to image. Using total internal reflection microscopy (TIRF) helps offset this problem, but clearly, more ways to minimize background noise and maximize photon output of fluorophores will be needed for maximizing resolution.

Fluorescent dyes generally have increased brightness and photostability compared to fluorescent proteins, but they have their own drawbacks. They cannot be genetically encoded and so must be targeted to the relevant protein using an antibody or other means. Antibody labeling is never very efficient and the large size of antibodies adds uncertainty to the position of the target molecule. Also, because antibodies do not permeate the plasma membrane, detergents are required to dissolve membranes for imaging molecules inside the cell and this can produce artifacts. Reversibly photoswitching probes such as cyanine dyes (used in STORM) have the further problem that once the molecule switches to a dark state, it can be reactivated and localized again. With repeated localization of the same molecule, the molecular distribution in the final highresolution image can be skewed and not representative of the true stoichiometry.

Sample-related problems in SR imaging must also be borne in mind. This includes artifacts that can arise from fixation, permeabilization and light exposure. The formaldehyde fixation typically used for immunofluorescence staining gives very poor ultrastructural preservation. To preserve ultrastructure in SR imaging, fixation conditions appropriate for electron microscopy are needed. Unfortunately, many of these conditions (using glutaraldehyde) can markedly diminish probe brightness and lifetime. Morphological changes due to fixation and permeabilization also need to be assessed by ultrastructural studies. Conversely, in live cell SR imaging the laser damage caused by the ultraviolet wavelengths typically used for photoactivation can become a problem.

In recent years, journals have instituted strict guidelines for the processing of images. As yet, it is not clear how SR images fall within these guidelines. In particular, PALM and STORM data in some important respects have more in common with graphs than with images. In a single image, color encodes both uncertainty and density of molecules, to create a map of the probability of finding a molecule at any position in space. Moreover, only select molecules will be rendered, according to how well they meet the criteria for being a 
single molecule (that is, the number of photons emitted) and how well they are localized. A standard way of rendering these images is necessary to simplify the interpretation of results. Similarly, standard criteria for the quality of the data should be met. Resolution requires not only that molecules are well localized, but also that they are dense enough to meet the Nyquist criterion ${ }^{14}$ so that the mean distance between molecules is less than half the desired resolution. Although none of these issues are so serious as to bring into question the utility of these new SR techniques, they do mean that careful treatment of the data is necessary to obtain biologically meaningful images.

It is impossible to foresee precisely which areas of biology will be most affected by SR fluorescence imaging, but there are several candidates that may undergo a significant transformation. These include cellular architecture (both static and dynamic), heterogeneous molecular organization, and dynamic protein assembly. A common feature of all of these areas is that they involve ensembles of molecules interacting to form large-scale cellular features, so that accessing information on specific proteins at the nanoscale could yield fundamentally new insights.

Functional cellular architecture requires cytoskeletal and accessory proteins to be organized in a precise manner. Furthermore, the structural organization of proteins can provide a clearer understanding of their roles, and lay the experimental groundwork for functional models of cellular machinery. Structural biology has made great progress in determining how pairs of molecules $(4-8 \mathrm{~nm}$ each) interact to build polymeric structures ( $>10 \mu \mathrm{m}$ long) such as microtubules, actin filaments and intermediate filaments. However, for structures such as the nuclear pore, centrosome, kinetochore, midbody or focal adhesions, which are complex three-dimensional assemblies formed by many interacting partners, a new approach is required. The goal is to achieve molecular resolution, such that individual molecules within a macromolecular assembly can be resolved and the stoichiometry of the proteins involved can be estimated. Further biological insight will be achieved with three-dimensional SR imaging, as well as live cell SR imaging to capture the dynamic remodeling of cellular architecture.

SR imaging may also open doors to better understanding molecular heterogeneity. The paradigm for protein organization in membranes has shifted from the classical, fluid mosaic model of random distributions to one in which lipid microdomains or cytoskeletal cages sequester or sort specific proteins. This heterogeneity is believed to underlie important functions-for example, in the Golgi, where cargo proteins and resident enzymes must interact but ultimately follow distinct itineraries. A variety of experimental tools, including immuno-electron microscopy and fluorescent resonance energy transfer (FRET), have been used to place limits on the sizes of membrane heterogeneities. Multicolor PALM ${ }^{15}$ provides a new way of looking at the organization of protein ensembles in the membrane, and of quantifying spatial correlations between different classes of proteins. Because of the single molecule information provided through PALM, the spatial relationship between proteins can be unambiguously defined and calculated as the probability of finding two interacting molecules within a set distance from each other. This line of inquiry extends beyond membrane proteins, and can be applied to many biological systems that may have nonrandom distributions, such as motor proteins on microtubules.

Finally, dynamic protein assembly may also be studied at the single-molecule level through SR imaging. Cellular responses to external signals begin at the plasma membrane, where the dynamic assembly of receptors can regulate cellular activity. Membrane-enveloped viruses, including $\mathrm{HIV}$, also assemble at the plasma membrane, exploiting mechanisms that have evolved for cellular trafficking. However, our physical model for how proteins assemble is far from complete. Although we know that the organization and dynamics of membrane proteins are heterogeneous, commonly used fluorescence-based measurements lack information at the molecular scale. Single molecule measurements have the opposite problem, as they are limited to looking at only a few molecules in a given cell and therefore lack information about molecular ensembles. Thus, the study of protein assembly has been limited by a lack of spatially resolved, dynamic information on ensembles of molecules. SR fluorescence imaging techniques combined with live cell imaging and single molecule tracking (sptPALM $)^{13}$ can address this limitation, and can thus help to determine how the dynamics of protein assembly are coordinated. Protein clusters from PALM imaging can be unequivocally defined by their local molecular density, and information on dynamic protein cluster statistics and morphology can help to elucidate assembly mechanisms and identify defects.

These are only three examples of how SR imaging may contribute to open biological questions. But they illustrate well how, from Leeuwenhoek's tentative hypotheses about the nature of life's basic constituents, we have now progressed to the point where SR imaging should allow biologists to witness individual molecules and their organization, the building blocks for all living things. Commercial versions of both STED and PALM are now available, and the SR imaging revolution is bound to spread from there. In the hands of creative biologists, this new extension of the human senses should help uncover many of nature's secrets.

1. Betzig, E., Trautman, J.K., Harris, T.D., Weiner, J.S. \& Kostelak, R.L. Science 251, 1468-1470 (1991).

2. Hell, S.W. \& Wichmann, J. Opt. Lett. 19, 780-782 (1994).

3. Gustafsson, M.G. Proc. Natl. Acad. Sci. USA 102, 13081-13086 (2005).

4. Betzig, E. et al. Science 313, 1642-1645 (2006).

5. Rust, M.J., Bates, J. \& Zhuang, X. Nat. Methods 3, 793-796 (2006).

6. Hess, S.T., Girirajan, T.P.K. \& Mason, M.D. Biophys. J. 91, 4258-4272 (2006).

7. Schermelleh, L. et al. Science 320, 1332-1336 (2008).

8. Willig, K.I., Rizzoli, S.0., Westphal, V., Jahn, R. \& Hell, S.W. Nature 440, 935-939 (2006).

9. Kittel, R.J. et al. Science 312, 1051-1054 (2006).

10. Huang, B., Wang, W., Bates, M. \& Zhuang, X. Science 319, 810-813 (2008).

11. Shroff, H., Galbraith, C.G., Galbraith, J.A. \& Betzig, E. Nat. Methods 5, 417-423 (2008).

12. Biteen, J.S. et al. Nat. Methods (in the press).

13. Manley, S. et al. Nat. Methods 5, 155-157 (2008).

14. Shannon, C.E. Proc. Inst. Radio Eng. 37, 10-21 (1949). Reprinted in Proc. IEEE 86, 447-457 (1998).

15. Shroff, H. et al. Proc. Natl. Acad. Sci. USA 104 20308-20313 (2007). 